\title{
Anabases
}

ANABASES Traditions et réceptions de l'Antiquité

17 | 2013

Varia

\section{Darstellung der Alterthums-Wissenschaft}

Friedrich August Wolf

\section{(2) OpenEdition}

Journals

Édition électronique

URL : http://journals.openedition.org/anabases/4229

DOI : 10.4000/anabases.4229

ISSN : 2256-9421

\section{Éditeur}

E.R.A.S.M.E.

\section{Édition imprimée}

Date de publication : 1 mars 2013

Pagination : 231-239

ISSN : 1774-4296

\section{Référence électronique}

Friedrich August Wolf, « Darstellung der Alterthums-Wissenschaft », Anabases [Online], 17 | 2013,

Online erschienen am: 01 April 2016, besucht am 14 November 2019. URL : http://

journals.openedition.org/anabases/4229 ; DOI : 10.4000/anabases.4229 
Anabases 17 (2013), p. 231-239.

\section{Darstellung der Alterthums-Wissenschaft ${ }^{1}$}

Friedrich August WolF

\section{Dédicace à Goethe}

Goethe,

Der Kenner und Darsteller des griechischen Geistes, empfange wohlwollend den mit Liebe dargebrachten Anfang einer Sammlung von Schriften und Aufsätzen, die bestimmt sind, hin und wieder das weite Gebäude von Kenntnissen aufzuklären, in welchen jener das Leben verschönernde Geist ursprünglich wohnte. An wen unter den Deutschen könnte man bei einem Unternehmen solcher Art [IV] eher denken, als an Den, in dessen Werken und Entwürfen, mitten unter abschreckenden modernen Umgebungen, jener wohlthätige Geist sich eine zweite Wohnung nahm? Seiner würden wir vor jedem andern gedenken, wäre auch nicht früher an einen der Herausgeber Sein freundschaftlicher Zuruf zu neuer veränderter Thätigkeit ergangen, ein Zuruf, der auch dem minder Vollendeten nachsichtigen Beifall gleichgesinnter Leser verhieß.

Doch nicht, um sich eines begünstigenden Genius unserer Litteratur zu versichern, wollten die Unternehmer dieser Zeitschrift ihr erstes Blatt mit Seinem Namen zieren. Dazu hätte es dieses öffentlichen Schmuckes nicht bedurft. Sie wollten bei einem so guten Anlasse der bildungsfähigen Jugend des Vaterlandes sagen, mit wie inniger Empfindung Derjenige zu ehren sei, der ihnen die hin $[\mathrm{v}]$ und her geworfene

1 Les textes présentés ici sont extraits de l'édition originale (Darstellung der AlterthumsWissenschaft, in F.A. Wolf, P. Buttmann (Hg.), Museum der Alterthums-Wissenschaft, Berlin, Realschulbuchhandlung, 1807), dont nous avons respecté la graphie et indiqué la pagination entre crochets. Nous remercions vivement Yannick Souladié pour ses remarques et ses conseils en vue de la présente traduction. 
Frage, zu welchem Ziele die Studien des Alterthums führen, schon längst genügender und schöner beantwortet hat, als die beste Erörterung je vermöchte. Denn woher ließ solche Erhebung über die engen Kreise und Tummelplätze des gewöhnlichen heutigen Lebens, woher ließen solche Ansichten von Welt und Kunst und Wissenschaft sich gewinnen, als aus dem innern Heiligthume der alterthümlichen Musenkünste, welches sich endlich einmal wieder in einem natürlich verwandten Gemüthe aufschloß? Einen Hypopheten von diesem Verdienst, der nicht allein die Sprüche und Ideen der verstummten Orakel auslegte, sondern selber viel Auslegungswürdiges hervorbrachte, näher erkennen und Seinen oft verborgenen Sinn ergründen zu lernen, schon dies wäre wohl des Schöpfens aus den ewigen Urquellen der Schönheit werth.

[vI] Ihr Wort und Ansehen, würdigster unserer Edeln, helfe hinfort uns kräftig wehren, daß nicht durch unheilige Hände dem Vaterlande das Palladium dieser Kenntnisse entrissen werde; wie wir denn gegründete Hoffnung hegen, daran ein unverlierbares Erbgut für die Nachkommen zu bewahren. Wo auch der Grund zu suchen sei, in der Natur unserer Sprache, oder in Verwandtschaft eines unserer Urstämme mit dem hellenischen, oder wo sonst etwa: wir Deutschen nach so manchen Verbildungen stimmen am willigsten unter den Neuern in die Weisen des griechischen Gesanges und Vortrages; wir am wenigsten treten zurück vor den Befremdlichkeiten, womit jene Heroën andern den Zutritt erschweren; wir allein verschmähen immer mehr, die einfache Würde ihrer Werke verschönern, ihre berühmten Unanständigkeiten meistern zu wollen. Wer aber bereits so viel [vII] von dem göttlichen Anhauche daheim empfand, dem wird der ernsthafte Gedanke schon leichter, in den ganzen Cultus der begeisternden Götter einzugehen.

Nur lassen Sie uns nicht weniger verhüten, daß zu diesen Orgien nicht das buntgemischte Volk ohne Vorbereitung und Andacht sich dränge, um mit dem Stabe der Begeisterung umherzutaumeln. Bewillkommen wir zwar manchen gern, der in unsern Kreisen Erheiterung und Labsal sucht nach dem Ernste strengerer Wissenschaften oder der Dürre blos erwerbsamer; ebenso denjenigen, der sich als eifrigen Liebhaber alles Schönen ankündigt; mag auch mancher der eigentlichen Genossen nicht gerade das Höchste der Wissenschaft nach den reichsten Gesichtspunkten umfassen, und sich mit einem und anderem Theile als fleißiger Arbeiter begnügen, immerhin befangen in dem Wahne, wirklich zu lieben, was [VIII] er nur als sein Tagwerk treibt: jedoch veredle jeder von Allen seine Bemühung und selbst sein Spielzeug durch sinnvolle Behandlung und durch die Richtung nach den anerkannten besten Zwecken. So werde, so bleibe der Deutsche, ohne die Emsigkeit des bloß gelehrten Sammlers zu verachten, ohne den bloßen Liebhaber allgemeiner Bildung zurückzuweisen, überall der tiefere Forscher und Ausleger des aus dem Alterthume fließenden Großen und Schönen; und er gebrauche solche Schätze, um unter dem Wechsel wandelbarer öffentlichen Schicksale den Geist seiner Nation zu befruchten, deren Bessere durch das Studium einheimischer Werke keinesweges unvorbereitet sind, die höhere Weihe zu empfangen.

Mögen Sie, Kenner und Darsteller des griechischen Geistes, noch lange ein wirksamer Beschützer und zufriedener Zuschauer so nützlicher Bestrebungen seyn! 
[IX] Möge Ihr geliebtes Weimar unter seinem herrlichen, von allen Musen gefeierten, Fürstenhause bald wieder in verjüngter Blüthe strahlend, auch fernerhin neue schöne Talente für das übrige Deutschland wecken! Möge Ihnen nie Kraft und ungestörte Muße fehlen, um auf dem Wege, worin Ihr Leben selbst dem Stufengange der Griechen nachahmt, itzt diese, itzt eine andere der holdesten Künste, und bald auch dunkele Räume oft entweihter Wissenschaften zu erleuchten!

\section{Préface de l'édition originale}

[3] Der Inhalt dieses Aufsatzes sollte seit geraumer Zeit als Einleitung zu einer Revision oder encyklopädischen Uebersicht derjenigen Kenntnisse dienen, die man gewöhnlich philologische nennt. Zwischen den Jahren 1783 und 1790, als der Verfasser auf einem damals berühmten Sitze deutscher Gelehrsamkeit zu lehren anfing, fühlte er sich ohne Unterlaß beunruhigt von dem Wunsche, sich selbst und seinen Zuhörern bestimmtere Rechenschaft zu geben über den allgemeinen Begriff, Gehalt, Zusammenhang und Hauptzweck jener Studien; über welches alles die Schriften der anerkanntesten Kenner wenig Befriedigendes darboten. Einige, die auf solche Betrachtungen sich beiläufig einließen, schwankten unentschlossen über die vornehmsten Tendenzen dieses ganzen Zweiges der Gelehrsamkeit, etwa wie gewisse Mitglieder [4] einer geheimen Gesellschaft, nicht immer Neulinge, einander über die Idee und Absicht ihres Ordens befragen; viele behaupteten, man erlerne die alten Sprachen der Geschichte und sogenannten Sachkenntnisse halber, wogegen die meisten das Lesen und Verstehen der Schriftsteller in den Grundsprachen als den letzten Zweck und die vielseitigste Beschäftigung mit den Sachkenntnissen als bloßes Mittel zum Verständnis der Schriftsteller betrachteten. Hie und da hörte man wol sogar urtheilen, überhaupt dürfte der Betrieb dieser Studien sich am besten durch die enge Verbindung rechtfertigen, worin sie nun einmal mit der gesammten Litteratur der Neuern, besonders mit manchen unserer nützlichsten und nutzbarsten Wissenschaften ständen, da freilich der erste Rang eigentlich solchen Kenntnissen gebühre, welche die Grundlagen des heutigen gesellschaftlichen Wohlstandes und aller bürgerlichen Glückseligkeit ausmachten: eine Ansicht, die manchem weisen Staatsmanne gefallen muß, der jede Wissenschaft gerade in dem Maaße schätzt, als sie zu den Künsten des Erwerbes im Frieden und im Kriege, zur Verbesserung der Finanzen, der Fabriken, des [5] Handels, der Land-und Haus-Wirthschaft beitragen mag $^{2}$.

Diese und andere von einander abweichende Meinungen verdoppelten des Verfassers Eifer, die höchsten Gesichtspunkte der alterthümlichen Philologie möglichst

2 Haec studia annonam non reddunt viliorem, soll einst jemand von den Sprachkenntnissen des Alterthums gesagt haben. Die Griechen hatten für dergleichen Denkart ein ausdrucksvolles Wort, das uns Neuern mangelt, vermuthlich weil uns die Idee lange fremd war, $\alpha \dot{\sigma} \sigma \rho \circ \kappa \varepsilon \rho \delta \varepsilon \imath$. 
genau zu erfassen, und einen Versuch zu machen, wie sich die einzelnen, theils auf deutschen Üniversitäten seit beinahe hundert Jahren erläuterten, theils noch in der Folge zu bearbeitenden, Doctrinen zu einem organischen Ganzen vereinigen ließen, um alles, was zu vollständiger Kenntniß des gelehrten Alterthums gehört, zu der Würde einer wohlgeordneten philosophisch-historischen Wissenschaft emporzuheben. Mit dieser Idee gab er in mehrmals wiederholten Vorlesungen unter dem Namen einer Encyklopädie und Methodologie der Studien des Alterthums einen Entwurf, dessen Ausführung nach erlangter Reife auch [6] dem größern Publicum mitgetheilt werden sollte. Was er damit bezweckte und auf welche Gegenstände die Vorträge, wie es schien, nicht ohne Nutzen lernbegieriger Jünglinge, gerichtet waren, drückt sich am deutlichsten durch die Worte selbst aus, in denen er die erste öffentliche Ankündigung davon machte: encyclopaedia philologica, in qua, orbe universo earum rerum, quibus litterae antiquitatis continentur, peragrato, singularum doctrinarum ambitus, argumenta, coniunctiones, utilitates, subsidia, denique recte et cum fructu tractandae cuinsque rationes illustrabuntur.

Eine erwünschte Lehrstelle gab dem Verfasser mehr als zwanzig Jahre hindurch Gelegenheit, sowohl die allgemeine Uebersicht der Wissenschaft des Alterthums als alle ihre wichtigern Theile vorzutragen, selbst bis auf die Litterär-Geschichte derselben und die historische Entwickelung des Ganges, den diese Studien bis auf unsere Zeit genommen haben. Begreiflich änderte und bildete sich bei jeder Wiederholung seines Vortrages der Gehalt und die Behandlungsweise der Materien; während aus den ersten noch sehr unvollkommenen [7] Skizzen durch den zu früh verstorbenen Fülleborn und andere Zuhörer ${ }^{3}$ eine Art von Grundrissen im Druck bekannt gemacht wurden. Ihn selbst hinderten von Zeit zu Zeit mehrere litterarische Arbeiten an der Ausführung seines Plans, überdem immer geneigt zu glauben, daß ein solches Werk nicht spät genug erscheinen könne, zumal wenn ein Gelehrter beständige Veranlassung hat, empfänglichen jungen Männern eine Anzahl leitender Begriffe und Aufschlüsse zu geben, die sie weiterhin noch besser als er selber ausbilden und mit eigenen Schätzen bereichern mögen. So kam er denn in den Fall, die Worte Quintilians auf sich anwenden zu dürfen: Quantum notando consequi potuerant, interceptum boni iuvenes, [8] sed nimium amantes mei, temerario editionis honore vulgaverant.

Noch sieht er unter den itzigen Umständen nicht der Muße entgegen, die sein Vorhaben begünstigen könnte. Unterdessen wurde es bei Anlaß der gegenwärtigen Zeitschrift nützlich erachtet, wenigstens die Grundzüge zu einer allgemeinen

3 G. G. Füllebornii Encyclopaedia philologica s. primae lineae Isagoges in antiquarum litterarum studia, Vratisl. 1798. 8. Edit. altera auct. et emendatior cur. J.G. Kaulfuß. Dieß ist ein Compendium nach den ersten Linien, die theils in Vorlesungen, theils auf einigen unvollendet gebliebenen Blättern : Antiquitäten von Griechenland, Halle, 1787, gegeben waren. Man vergleiche unseres J.E. Koch's Hodegetik für das Universitäts-Studium, Berlin 1792, 8. S. 64-98. und Desselben Encyklopädie aller philologischen Wissenschaften, Berlin, 1793. 8. 
Darstellung der griechischen und römischen Alterthums-Kenntnisse zu liefern, doch nicht lateinisch, wie das größere Buch geschrieben werden sollte, sondern in der ihm ungewohntern Sprache, die auch unter unsern Humanisten mehrere Leser findet. Die Ausarbeitung sollte itzt das Geschäft weniger Wochen seyn, und wurde anziehend durch die Entfernung von den Drangsalen der Zeit, die uns mahnen, in angenehmern Perioden der Geschichte, vorzüglich des schönen Alterthums, Erhohlung und frische Energie zu suchen ${ }^{4}$.

[9] Ob übrigens unsere Behandlung der Gegenstände und die Angabe ihrer Zwecke den Namen einer Wissenschaft, der hier diesen Kenntnissen ertheilt wird, hinlänglich rechtfertige, mögen andere beurtheilen. Aber hoffentlich wird niemand unter den Gelehrten, die sich mit Natur-Kenntnissen und den genauern Wissenschaften beschäftigen, von der Natur, in ihrer echten großen Bedeutung, den Menschen ausschließen; und niemand, der unsere Studien ein wenig kennt, wird glauben, daß das, was durch historische Untersuchungen des Alterthums und durch Bekanntschaft mit den Sprachen und den unsterblichen Werken desselben zur harmonischen Ausbildung des Geistes und Gemüths gewonnen wird, eben so vollkommen auf irgend einem andern Wege könne erreichet werden. Berlin, im Jul. 1807.

\section{Dédicace à Goethe}

Goethe,

Le connaisseur et le représentant de l'esprit grec, accueille-t-il avec bienveillance les débuts, offerts avec amitié, d'une collection d'écrits et d'essais destinés à éclairer çà et là le vaste édifice de conceptions parmi lesquelles vivait originellement cet esprit qui embellit l'existence.

À qui parmi les Allemands eût-on pu penser au seuil d'une telle entreprise sinon à celui dans les œuvres et les projets duquel cet esprit bienfaiteur, dans un environnement moderne décevant, prit une seconde demeure ? À lui, avant tout autre, nous eussions pensé quand bien même son invitation amicale à une activité nouvelle et transformée ne fût pas parvenue au préalable à l'un des deux éditeurs, invitation qui promettait, même à ce qui est moins accompli, les suffrages indulgents de lecteurs pareillement disposés.

Pourtant, ce n'était pas pour s'assurer d'un génie favorable de notre littérature que les fondateurs de cette revue voulurent que la première page de celle-ci fût ornée de son nom : pour cela, il n'eût pas été besoin de cet hommage public. Ils voulurent,

4 Gewißer Leser wegen mag hier die Bemerkung stehen, daß bei dieser Schrift nichts von dem benutzt wurde, was etwa in den letztern Jahren über das Allgemeine der philologischen Studien herausgekommen ist. Zu Lesen und Nachschlagen von Büchern war überhaupt weder Veranlassung, noch Muße, noch Neigung; außer wenn zuweilen zufällige Erinnerung dazu verleitete. 
en une occasion si propice, dire à la jeunesse cultivée de la patrie avec quel sentiment profond honorer celui qui depuis longtemps a répondu, de façon plus satisfaisante et plus élégante que n'aurait pu le faire la meilleure explication, à la question, adressée à eux de temps à autre, du but auquel conduit l'étude de l'Antiquité.

Car d'où une telle capacité à s'élever au-dessus des cercles étroits et des terrains de jeu favoris de l'existence ordinaire d'aujourd'hui, d'où de telles vues sur le monde, l'art et la science furent-elles atteintes, sinon depuis le sanctuaire intime des antiques arts des Muses, qui s'ouvrit finalement, une fois encore, dans une âme naturellement parente ? Connaître de plus près un hypophète d'un tel mérite, qui non seulement sut interpréter les arrêts et les idées de l'oracle devenu muet, mais produisit lui-même bien des paroles dignes d'être interprétées, apprendre à sonder son esprit parfois obscur : cela déjà n'en reviendrait pas moins à puiser à l'éternelle source première de la beauté.

Puissent votre parole et votre prestige, vous le plus digne d'entre nos grands, aider désormais à ce que nous refusions vigoureusement que le palladium de ces savoirs soit arraché à la patrie par des mains impies, comme nous nourrissons l'espoir légitime de préserver ainsi, pour les générations à venir, un héritage impérissable. Nous, Allemands - où qu'il faille du reste chercher les origines de ce phénomène, dans la nature de notre langue ou dans la parenté de l'une de nos souches primitives avec celle des Grecs, ou ailleurs encore -, sommes, après tant de déformations, les plus prompts parmi les Modernes à nous accorder aux modes du chant et de la déclamation des Grecs; moins que tous nous cédons devant ces étrangetés par lesquelles ces héros se rendent difficiles d'accès aux autres; seuls, nous nous abstenons toujours plus de vouloir enjoliver la digne simplicité de leurs ouvres et dompter leurs fameuses indécences. À celui qui reçut chez lui tant de souffle divin paraitra plus légère encore l'idée grave de prendre part au culte tout entier des dieux enivrants.

Ne nous permettez pas moins, toutefois, de veiller à ce que le peuple bigarré ne se presse à ces orgies sans préparation et sans dévotion, pour s'y perdre sous le coup de l'ivresse. Souhaitons bien volontiers la bienvenue à celui qui dans nos cercles cherche le divertissement et la fraîcheur après le sérieux des sciences plus dures ou l'aridité de sciences simplement plus lucratives; la bienvenue, aussi, à celui qui se présente comme un amateur zélé de tout ce qui est beau. Il se peut aussi que tel de nos collègues ne se risque pas à embrasser, à partir des points de vue les plus riches, le sommet de la science, et se contente de ne cultiver, en ouvrier zélé, que l'une ou l'autre de ses parties, cependant toujours victime de l'illusion d'aimer véritablement ce qu'il n'exerce que comme sa tâche quotidienne : qu'il anoblisse pourtant son effort et même son passetemps par un traitement raisonné et orienté dans la direction des buts reconnus comme les meilleurs. Puisse l'Allemand ainsi devenir et continuer à être en tout, sans mépriser le zèle du simple collectionneur érudit et sans éconduire le simple amateur de culture générale, le plus profond chercheur et exégète de la grandeur et de la beauté issues de l'Antiquité ; et qu'il utilise ces trésors, dans les changements qui affectent les destinées communes, pour féconder l'esprit de sa nation, dont les membres les meilleurs, grâce à 
l'étude des œuvres de leur patrie, ne sont nullement impréparés à recevoir cette consécration suprême.

Puissiez-vous donc, connaisseur et représentant de l'esprit grec, être longtemps encore le puissant protecteur et l'heureux témoin de ces efforts si précieux! Puisse votre chère Weimar, rayonnant bientôt dans une floraison nouvelle sous la splendeur de sa maison princière, célébrée par toutes les Muses, continuer à éveiller de beaux talents pour le reste de l'Allemagne! Puissiez-vous, sur le chemin où votre vie même imite la marche des Grecs, ne manquer jamais de force et de paisible loisir pour éclairer ici l'un des arts les plus gracieux, là un autre, bientôt enfin les sombres pièces de sciences volontiers profanes!

\section{Préface de l'édition originale}

Le contenu de cet essai devait depuis longtemps servir d'introduction à un inventaire ou à un exposé encyclopédique des savoirs que l'on appelle d'ordinaire philologiques. Entre 1783 et 1790, tandis que l'auteur commençait à enseigner dans un lieu naguère célèbre de l'érudition allemande, il se sentit assailli sans répit par le désir de faire, pour ses auditeurs comme pour lui-même, une mise au point sur le concept général, la teneur, le cadre et le but principal de ces études - sur quoi les écrits des spécialistes les plus reconnus n'offraient que peu de choses satisfaisantes. Quelques-uns de ceux qui s'étaient occupés incidemment de ces questions s'interrogeaient avec hésitation sur les tendances les plus notables de toute cette branche de l'érudition, un peu comme certains membres d'une société secrète, et pas seulement ses néophytes, se consultent sur l'idée et le dessein de leur ordre. Nombre d'entre eux prétendaient que l'on n'apprend les langues anciennes qu'en vue de l'histoire et des savoirs factuels, tandis que la plupart, au contraire, regardaient la lecture et la compréhension des auteurs en langue originale comme le but ultime, et l'étude compréhensive des savoirs factuels seulement comme un moyen en vue de la compréhension des auteurs. On entendait même dire çà et là que la pratique de ces études se justifiait le mieux par le lien étroit qui les unit à toute la littérature académique des Modernes, en particulier à bon nombre de nos sciences les plus utiles et les plus profitables - étant entendu en effet que la première place revenait à ces savoirs qui constituent les fondements de la prospérité commune d'aujourd'hui et de la félicité bourgeoise. Un point de vue qui devrait plaire à maints sages hommes d'État, qui n'apprécient une science que dans la mesure où elle est susceptible de contribuer à l'art d'acquérir, en temps de paix comme en temps de guerre, à l'amélioration des finances, des usines, du négoce, de l'économie domestique et de l'agriculture ${ }^{5}$.

5 Haec studia annonam non reddunt viliorem, aurait dit quelqu'un, un jour, des connaissances linguistiques sur l'Antiquité. Les Grecs avaient pour désigner cette façon de penser un 
L'une et l'autre de ces opinions divergentes ne firent que redoubler le zèle de l'auteur pour saisir, de façon aussi précise que possible, les aspects les plus élevés de la philologie classique, et tenter de voir comment les doctrines particulières, fussent-elles cultivées depuis près d'un siècle dans les universités allemandes ou encore à élaborer, pouvaient être réunies en un tout organique. Cela afin que tout ce qui relève d'une connaissance complète de l'Antiquité savante soit élevé à la dignité d'une science philosophico-historique bien ordonnée. Animé par cette idée, l'auteur proposa dans les leçons qu'il donna régulièrement, sous le nom d'" Encyclopédie et méthodologie de l'étude de l'Antiquité ", une esquisse dont le produit devait être partagé, quand il serait plus mûr, avec un public plus large. Ce qu'il visait par là et sur quels objets le propos devait porter - non sans quelque profit, semblait-il, pour la jeunesse avide d'apprendre - s'exprime le plus nettement dans les mots mêmes dans lesquels il fit de ce projet la première présentation publique : Encyclopaedia philologica, in qua, orbe universo rerum, quibus litterae antiquitatis continentur, peragrato, singularum doctrinarum ambitus, argumenta, coniunctiones, utilitates, subsidia, denique recte et cum fructu tractandae cuiusque rationes illustrabuntur.

La chaire de son vœu donna à l'auteur, pendant plus de trente ans, l'opportunité de présenter tant une vue générale de la science de l'Antiquité que ses parties principales, jusques et y compris l'histoire de l'érudition et le cours historique que ces études prirent jusqu'à notre époque. Naturellement, la teneur et le traitement de la matière furent modifiés et façonnés au gré des leçons ; cependant qu’à partir des premières esquisses, encore très inachevées, une manière de précis fut publiée par le regretté Fülleborn et d'autres auditeurs ${ }^{6}$. Des travaux d'érudition, de temps à autre, empêchèrent par ailleurs l'auteur de mener à bien son projet, bien qu'il fût toujours enclin à croire qu'un tel ouvrage ne devait pas paraitre trop tard. Cela d'autant plus qu'un érudit est en permanence à même de communiquer à de jeunes gens réceptifs maintes notions et explications fondamentales, qu'ils peuvent ensuite développer mieux encore que lui-même ne l'a fait et enrichir de leurs propres trésors. Il eut alors l'occasion de pouvoir s'appliquer à lui-même le mot de Quintilien : Quantum notando consequi potuerant, interceptum boni iuvenes, sed nimium amantes mei, temerario editionis honore vulgaverant.

L'auteur n'envisage pas encore, dans les circonstances actuelles, le loisir qui puisse favoriser son projet. En attendant, il a été jugé utile, à l'occasion de la parution de la

mot expressif, qu'il nous manque à nous Modernes, probablement parce que l'idée nous

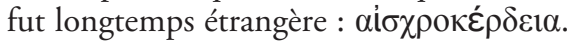

6 G. G. Füllebornii Encyclopaedia philologica s. primae lineae Isagoges in antiquarum litterarum studia, Vratisl. 1798. 8. Edit. altera auct. et emendatior cur. J.G. Kaulfuß. Il s'agit d'un compendium, d'après les premiers éléments, donnés à partir soit de leçons, soit de quelques feuillets restés inachevés : Antiquitäten von Griechenland, Halle, 1787. Comparer avec notre J.E. Koch's Hodegetik für das Universitäts-Studium, Berlin 1792, 8. p. 64-98 et, du même, Encyklopädie aller philologischen Wissenschaften, Berlin, 1793. 8. 
présente revue, de proposer à tout le moins les traits fondamentaux d'une présentation générale des connaissances sur l'Antiquité grecque et romaine, non en latin, toutefois, ainsi que devait être écrit le livre plus important qu'il projetait, mais dans une langue pour lui plus inhabituelle, qui parmi nos humanistes trouve pourtant nombre de lecteurs. La composition ne devait plus être désormais que l'affaire de quelques semaines, et fut rendue agréable par l'éloignement d'avec les tourments du temps, qui nous incitent à chercher dans des périodes plus douces de l'histoire, de préférence dans la belle Antiquité, du répit et une énergie nouvelle?

D'autres pourront juger si le traitement de la matière et l'exposé de ses buts justifient suffisamment le nom de science donné ici à ces connaissances. Pourvu cependant que personne parmi les érudits qui s'occupent des savoirs sur la nature et des sciences exactes n'exclue l'homme de la nature, au sens véritable et noble du terme ; et que personne, parmi ceux qui savent quelque peu de nos études, ne croie que ce que l'on acquiert par les recherches sur l'Antiquité ainsi que par la connaissance de ses œuvres immortelles et des langues anciennes, en vue de l'harmonieuse formation de l'esprit et du cœur, puisse être atteint aussi parfaitement par quelque autre chemin que ce soit. Berlin, juillet 1807.

7 À l'usage de certains lecteurs, on peut observer que pour cet écrit rien n'a été utilisé de ce qui a paru ces dernières années dans le domaine général des études philologiques. Pour la lecture et la consultation d'ouvrages n'étaient généralement ni le motif, ni le loisir, ni l'inclination, hormis quand parfois un souvenir fortuit y incitait. 